\title{
Surveillance of Human Papilloma Virus in the United States to Evaluate Vaccine Impact
}

\author{
Andrew Wilson*1, Ryan Welch ${ }^{2}$ and Rosemary She ${ }^{3}$ \\ 'Department of Family and Preventive Medicine, University of Utah, Salt Lake City, UT, USA; ${ }^{2}$ ARUP Institute for Clinical and \\ Experimental Pathology, Salt Lake City, UT, USA; ${ }^{3}$ University of Southern California Keck Medical Center, Los Angeles, CA, USA
}

\section{Objective}

To establish and evaluate an HPV surveillance protocol and determine the rate of change of high-risk HPV in the United States since 2004 using archived reference laboratory data.

\section{Introduction}

Numerous studies have demonstrated a causal relationship between human papillomavirus (HPV) and cervical cancer ${ }^{1}$. By 2007 two vaccines targeting HPV were available in the United States. Both vaccines have shown close to $100 \%$ efficacy against HPV types 16 and 18 , the cause of $70 \%$ of all cervical cancers ${ }^{2}$. It is hypothesized that with routine vaccination the prevalence of HPV and HPV-associated cancers should decline ${ }^{3}$.

A need exists for surveillance with national coverage ${ }^{2}$. The purpose of this study is to present a method to estimate rate of change of high-risk HPV in the United States since 2004 in women using national reference laboratory data.

\section{Methods}

This study was approved by the University of Utah Institutional Review Board (IRB \#00039866). Data from patient samples submitted to ARUP Laboratories (Salt Lake City, UT) for HPV testing by the Digene ${ }^{\circledR}$ hc2 HPV DNA Test are archived in an electronic data warehouse. These data were then queried producing 856,836 records for women age 14-60 with a conclusive positive or negative result between January 1, 2004 and June 1, 2013.

Positivity rates and exact binomial confidence intervals were calculated by age strata and race by year. Generalized linear models were created to assess differences in positivity between age categories and race, and assess changes over time.

Finite mixture models were used to investigate patterns within the distribution of time between sample collection and time of final result for all patients ${ }^{4}$. Differences in time between collection and reporting indicate different ordering patterns, which were used to reduce potential bias ${ }^{5}$. We also compared positivity rates between these different testing patterns.

\section{Results}

Unadjusted positivity rates for the high-risk HPV group was $27.2 \%$ for all age groups combined. Highest rates occurred in individuals aged 14-19 and Hispanics. Data from 48 states and 692 sites were represented.

While the positivity rate decreased for all age groups from 2004 to 2013, the higher age categories showed less of a downward trend following vaccine introduction, and the two age categories 20-24 and 25-29 showed a significantly different downward trend between pre and post-vaccine time periods.

\section{Conclusions}

Using retrospective data from a national reference lab from 2004 to 2013, the positivity estimate of high-risk HPV in all age groups is $27.2 \%$. Based on our national data from patients with access to healthcare, we show that there was a significant downward trend in positivity rates for all age categories over time. However, contrary to the other age groups, women 20-24 and 25-29 years old had no significant decrease in positivity rate prior to introduction of vaccine (2004-2006). In fact, women in these two age categories saw a faster rate of decline in HPV positivity rates post-vaccine.

Models have predicted that the introduction of a vaccine should have a strong (30-44\% decrease) impact on HPV positivity in the types covered by the vaccine ${ }^{3}$. Our data shows more modest effects ( $8 \%-20 \%$ decrease, depending on strata), which may be a combination of insufficient vaccination coverage or a reflection of our data containing a mixture of vaccine-preventable and other HPV strains.

\section{Keywords}

Human Papilloma Virus; Prevalence; Surveillance; Vaccine; Gardasil

\section{Acknowledgments}

We'd like to thank Anna Gardiner, Brian Shirts, and Lindsay Larkin Wilson for their input.

\section{References}

1. Lowy DR, Schiller JT. Prophylactic human papillomavirus vaccines. J Clin Invest. May 2006;116(5):1167-1173.

2. Centers for Disease C, Prevention. Human papillomavirus-associated cancers - United States, 2004-2008. MMWR. Morbidity and mortality weekly report. Apr 20 2012;61:258-261.

3. Hughes JP, Garnett GP and Koutsky K. The Theoretical Population-Level Impact of a Prophylactic Human Papilloma Virus Vaccine. Epidemiology 2002;13:631-639.

4. DAY NE. Estimating the components of a mixture of normal distributions. Biometrika. December 1, 1969 1969;56(3):463-474.

5. Shirts BH, Jackson BR. Informatics methods for laboratory evaluation of HPV ordering patterns with an example from a nationwide sample in the United States, 2003-2009. Journal of pathology informatics. 2010;1:26.

\footnotetext{
*Andrew Wilson

E-mail: wilson.stats@gmail.com
} 